\title{
Pretreatment with interleukin 35-engineered mesenchymal stem cells protected against lipopolysaccharide-induced acute lung injury via pulmonary inflammation suppression
}

\author{
Xiaoning Zhang ${ }^{1,2} \cdot$ Zhiqiang Zhang $^{3} \cdot{\text { Mingyan } \mathrm{Ju}^{1} \cdot \text { Jiaci Li }^{1} \cdot \text { Yaqing Jing }}^{1} \cdot$ Yuxia Zhao ${ }^{1} \cdot$ Chao $\mathrm{Gu}^{1} \cdot \mathrm{Ming}$ Dong ${ }^{4}$. \\ Guang $\mathrm{Li}^{1} \cdot \mathrm{Yi} \mathrm{Liu}^{1}$ (1)
}

Received: 15 October 2019 / Accepted: 17 February 2020 / Published online: 13 March 2020

(c) Springer Nature Switzerland AG 2020

\begin{abstract}
Acute lung injury (ALI)-triggered pulmonary injury has been associated with high mortality, despite advances in drug treatment and supportive therapy. Remarkable progress has been made in attenuating the inflammatory injury associated with ALI using mesenchymal stem cells (MSCs)-based cell and gene therapy. However, to date, the benefits of interleukin-35 (IL-35)-modified MSCs in ALI intervention have not been investigated. In the present study, adult male C57BL/6 mice randomly received intravenous infusion of adipose-derived mesenchymal stem cells (ADSCs) constitutively expressing IL-35 (IL-35-GFP-ADSCs) or GFP (GFP-ADSCs) via retrovirus-mediated transduction $\left(8 \times 10^{5}\right.$ cells per mice) or isotonic saline 7 days before ALI modeling to investigate the effect and related mechanism. ALI was performed by lipopolysaccharide (LPS) inhalation for $24 \mathrm{~h}$. Normal mice served as the sham group. The results indicated that compared with GFP-ADSCs, IL-35-modified ADSCs significantly increased cellular and pulmonary IL-10 and IL-35 production. Pretreatment with IL35-ADSCs markedly reduced body weight loss, pulmonary wet/dry weight ratio and pathological injury. $\mathrm{The} \mathrm{PO}_{2}$ was rescued to normal levels in mice that received IL-35-ADSCs. IL-35-ADSCs infusion apparently inhibited IL-6 release, protein leakage and MPO activity but greatly elevated IL-35 level in the bronchoalveolar lavage fluid (BALF). Splenic regulatory T cells in IL-35-ADSCs-pretreated mice got effective increase. Moreover, IL-35-ADSCs pretreatment remarkably inhibited neutrophil and macrophage infiltration and greatly decreased IL-6, tumor necrosis factor $\alpha$ (TNF- $\alpha$ ) and Toll-like receptor 4 (TLR4) expression. In conclusion, pretreatment with IL-35-engineered ADSCs provided effective protection against LPSinduced ALI through suppression of pulmonary inflammation and, thus, might be a promising strategy to improve outcomes after ALI. The enhanced paracrine and immunosuppressive capacity of IL-35-ADSCs might contribute to their beneficial effects. However, further studies are needed to illuminate the detailed mechanism.
\end{abstract}

Keywords Acute lung injury (ALI) $\cdot$ IL-35 · Adipose-derived mesenchymal stem cells (ADSCs) $\cdot$ Inflammation $\cdot$ Gene modification

Yi Liu

xiaoyurly@163.com

1 Department of Genetics, School of Basic Medical Sciences, Tianjin Medical University, 22 Qixiangtai Road, Heping

District, Tianjin 300070, People's Republic of China

2 Institute of Basic Medicine, Shandong Provincial Hospital Affiliated to Shandong First Medical University, Jinan 250012, People's Republic of China

3 Department of Pathology, Tianjin Hospital of ITCWM, Nankai Hospital, Tianjin 300100, People's Republic of China

4 Department of Lung Cancer Surgery, Tianjin Medical University General Hospital, Tianjin 300052,

People's Republic of China

\section{Introduction}

Acute lung injury (ALI) or its severe form, acute respiratory distress syndrome (ARDS), is the major cause of acute respiratory failure in critical patients (Cho et al. 2016; Cobbold et al. 2003). It is characterized by progressive hypoxemia and increased lung permeability, and the pathophysiologic events in ALI/ARDS include disruption of the microvascular endothelial-alveolar epithelial barrier, pulmonary edema, uncontrolled inflammation and respiratory failure (Matthay et al. 2012; Ware and Matthay 2000). Despite the progress in pharmacotherapy and supportive treatments, such as corticosteroids, statins, lung 
protective ventilation strategy (LPVS) and extracorporeal membrane oxygenation (ECMO), ALI/ARDS still presents with high morbidity (Bellani et al. 2016; Fanelli and Ranieri 2015; Matthay et al. 2012; Monge et al. 2014; Ware and Matthay 2000).

The common causes of ALI/ARDS may include trauma, tissue damage, infection, and shock (except for cardiogenic shock), making it a highly heterogeneous disease (Manabe et al. 2018; Matthay et al. 2012). Apart from the complex pathogenesis, the consensus among researchers is that suppression of the respiratory burst and overwhelming pulmonary inflammation is the key target for ALI/ARDS treatment (D'Alessio et al. 2009; Lin et al. 2018; Williams and Chambers 2014).

Recently, the progress in mesenchymal stem cells (MSCs)-based cell and gene therapy for ALI/ARDS treatment has received much attention. Many investigations have confirmed the benefits of MSCs in lipopolysaccharide (LPS)-induced ALI therapy, mainly due to their immunomodulation capacity and secretion of anti-inflammatory cytokines ( $\mathrm{Li}$ et al. 2016; Shi et al. 2010). Modification of certain anti-inflammatory genes, such as IL-10 (Wang et al. 2018a), nuclear factor erythroid-2 related factor 2 (Nrf2) (Zhang et al. 2018b) and heme oxygenase (HO-1) (Chen et al. 2019), can further enhance the immunosuppressive potential of MSCs and achieve better outcomes in ALI intervention. However, most of these findings were discovered based on cells administration post ALI modeling; the protective effects of MSCs pretreatment have not been well described.

Interleukin-35 (IL-35), composed of Epstein-Barr virus-induced gene 3 (EBi3) and the IL-12 p35 subunit (IL-12A), is a newly identified immunosuppressive cytokine in the IL-12 family (Collison et al. 2007). The anti-inflammatory activity of IL-35 has been verified in inflammation, infection and other immune-related disorders (Kuo et al. 2011; Maddaloni et al. 2018; Wang et al. 2018c). Wang et al. reported that IL-35 can protect against clinical and experimental models of ARDS (Wang et al. 2019). Thus, IL-35 genetically engineered MSCs is a promising option for ALI therapy. However, the efficacy of IL-35-engineered ADSCs against ALI has not yet been examined.

The present study focused on the protective effect of IL-35-engineered MSCs on LPS-induced ALI. We chose adipose-derived mesenchymal stem cells (ADSCs), whose proliferation, differentiation and immunomodulation potential are similar to that of MSCs from other sources (Ozdemir et al. 2019), to perform gene modification. Because the inflammatory microenvironment is challenging for cell survival, IL-35-modified ADSCs were administered 7 days before LPS exposure. Then, their protective effect on pulmonary injury and the latent mechanism were explored.

\section{Materials and methods}

\section{Animals}

Adult male specific-pathogen-free (SPF) C57BL/6 mice (aged 8-10 weeks, weighing $23 \pm 2 \mathrm{~g}$ and aged 6 weeks, weighing $15 \pm 2 \mathrm{~g}$ ) were purchased from the Laboratory Animal Center of the Academy of Military Medical Science (Beijing, China). Mice were maintained under SPF conditions. Experimental protocols and animal care methods were approved by the Animal Care and Use Committee of Tianjin Medical University (approval number TMUaMEC2017012 and approval date 20/9/2017). All experiments were conducted according to the standard guidelines of the Animal Care and Use Committee of Tianjin Medical University.

\section{Cells}

The packaging cell line HEK293T is a kind gift from Prof. Deling Kong (Nankai University, Tianjin). Cells were routinely cultured in high-glucose DMEM (Gibco, USA) supplemented with $10 \%$ fetal bovine serum (FBS, Gibco, USA) and $5000 \mathrm{U} / \mathrm{mL}$ penicillin/streptomycin (PS, Gibco, USA). Adipose-derived mesenchymal stem cells (ADSCs) were isolated from 6-week-old mice as described previously (Yao et al. 2015). In brief, cells from adipose tissues near the groin and epididymis were adherently cultured in $\alpha$ MEM (Gibco, USA) containing 20\% FBS and 5000 $\mathrm{U} / \mathrm{mL}$ PS. The culture medium was replaced every three days until the cell confluence reached approximately $90 \%$. The 3rd passage cells identified by fluorescence activated cell sorting (FACS) after staining of surface markers were used for gene modification. Briefly, about $1 \times 10^{5}$ ADSCs per sample were resuspended in 50- $\mu \mathrm{L}$ PBS and incubated with $2-\mu \mathrm{L}$ phycoerythrin (PE)-conjugated antibodies $\mathrm{CD} 29$ and CD44 (eBioscience, USA), fluorescein isothiocyanate (FITC)-conjugated CD45 (eBioscience, USA) and CD90.1 (eBioscience, USA) antibodies separately for $30 \mathrm{~min}$ at $4{ }^{\circ} \mathrm{C}$ in the dark. Then, the cell suspension was centrifuged and subsequently washed with PBS and fixed by $4 \%$ paraformaldehyde (PFA). The staining positive cells were detected by a flow cytometer (BD FACS Calibur, USA) and cells without any label served as negative control. Data were analyzed by FlowJo software (version 7.6.1, USA).

\section{Retrovirus production and transfection}

The retroviral vector pMSCV-IL-35-GFP reported in our previous study carried the murine $I L-35$ fragment covering Ebi3 linked with $I l 12 a$ (Zhang et al. 2018c). One day before transfection, HEK293T cells were plated, and the cell fusion 
rate was approximately $80 \%$ after $24 \mathrm{~h}$. HEK293T cells were transfected using FuGENE ${ }^{\circledR}$ HD Transfection Regent (Promega, USA) according to the manufacturer's instructions. Specifically, the retroviral vector pMSCV-IL-35-GFP or pMSCV-GFP combined with another two helper plasmids and FuGENE were dissolved in equal volume of Opti-MEM (Invitrogen, USA) medium, respectively. Then, the plasmids and transfection reagent were mixed for $20 \mathrm{~min}$ at room temperature. The mixture was evenly added to HEK293T cells maintained in 5-mL Opti-MEM medium and incubated in $5 \% \mathrm{CO}_{2}$ at $37{ }^{\circ} \mathrm{C}$. After $6 \mathrm{~h}$, another 5-mL fresh DMEM medium containing $10 \%$ FBS was supplemented and the cells were continued to be cultured for $48 \mathrm{~h}$. The supernatant rich in retrovirus particles was collected, followed by centrifugation at $1500 \times g$ for $10 \mathrm{~min}$ and filtering through a $0.45-\mu \mathrm{m}$ filter. Then, the filtrates were condensed at 10:1 ratio using a $100-\mathrm{kDa}$ evaporating column (Millipore, USA). ADSCs at the 3rd passage were exposed to $1-\mathrm{mL}$ of concentrated IL-35-GFP or GFP virus in 2-mL of medium for $6 \mathrm{~h} \mathrm{in} 10-\mathrm{cm}$ cell culture dishes in the presence of $8 \mu \mathrm{g} / \mathrm{mL}$ polybrene (Sigma-Aldrich, USA) and were expanded to the 4th passage. The transfection efficiency of ADSCs modified by IL-35 (IL-35-ADSCs) or GFP (GFP-ADSCs) and the exogenous gene expression were determined via FACS and quantitative real-time PCR (qPCR) before cell injection. In addition, the culture medium of IL-35-ADSCs and GFP-ADSCs was collected $48 \mathrm{~h}$ post transfection to detect the concentration of IL- 10 .

\section{LPS-induced ALI in mice}

Eighteen mice (8-10 weeks) were anesthetized via isoflurane inhalation in a small sealed box and randomly received $8 \times 10^{5} \mathrm{IL}-35-\mathrm{ADSCs}$ (in $200-\mu \mathrm{L}$ sterile normal saline (NS)), $8 \times 10^{5}$ GFP-ADSCs (in $200-\mu \mathrm{L} \mathrm{NS}$ ) or isovolumetric NS via tail vein injection (6 mice per group) 7 days before LPS administration. Another 6 mice received nothing and were used as the sham group. The LPS-induced ALI model was constructed in mice in accordance with previous reports (Zhang et al. 2018b). All 18 mice received intratracheal (i.t.) instillation of a single dose of 100- $\mu \mathrm{g}$ LPS (Escherichia coli serotype 0111: B4, Sigma-Aldrich) in 50- $\mu$ L NS. Mice in the sham group were administered 50- $\mu$ L NS instead of LPS. The four groups were named Sham, saline + LPS (negative control), GFP-ADSCs + LPS (positive control) and IL35-ADSCs + LPS, respectively. All mice were anesthetized through intraperitoneal injection of chloral hydrate $(330 \mathrm{mg} /$ $\mathrm{kg}$ ), and bronchoalveolar lavage fluid (BALF) and abdominal aortic blood were collected $24 \mathrm{~h}$ post LPS exposure. Then, the mice were killed via cervical dislocation, and lung, spleen and peripheral blood samples were harvested. The weight of the mice was recorded at different time points. The wet/dry weight ratio of the right lung was also calculated.

\section{Blood gas analysis}

Fresh abdominal aortic blood samples under heparinization were used immediately to perform blood gas analysis. The oxygen pressure $\left(\mathrm{PO}_{2}\right)$ and carbon dioxide pressure $\left(\mathrm{PCO}_{2}\right)$ in each mouse were tested using a Blood Gas Electrolyte Analyzer (TOP-C1072, Guangzhou JinYuan Medical Equipment Co., Ltd., China).

\section{Enzyme-linked immunosorbent assay (ELISA)}

The IL-10 content in the culture medium of GFP-ADSCs and IL-35-ADSCs was measured via ELISA using the corresponding kit (Biolegend, USA) according to the protocol provided by the manufacturer. The concentration of IL-35 and IL-6, the total protein, and myeloperoxidase (MPO) activity in BALF were also measured using ELISA kits (Biolegend, USA). Mice were fasted for approximately $8 \mathrm{~h}$ before serum collection. The ELISAs were performed in triplicate for each sample.

\section{FACS analysis of splenic Tregs}

The $\mathrm{CD} 4{ }^{+} \mathrm{CD} 25^{+}$Tregs among splenic lymphocytes were identified via FACS analysis. Briefly, after removal of most red blood cells using erythrocyte lysis buffer, approximately $1 \times 10^{6}$ cells were incubated with anti-mouse fluorescein isothiocyanate (FITC)-CD4 (eBioscience, USA) and antimouse phycoerythrin (PE)-CD25 (Biolegend, USA) antibodies. After incubation at $4{ }^{\circ} \mathrm{C}$ for $30 \mathrm{~min}$, the cells were washed with staining buffer and centrifuged at $2000 \mathrm{rpm}$ for $5 \mathrm{~min}$. The positively stained cells were assayed with a flow cytometer. FlowJo 7.6.1 software was utilized for subsequent data analysis.

\section{Histological and immunohistochemical staining}

Lung samples were fixed with $4 \%$ paraformaldehyde, embedded in paraffin and cut into 5- $\mu \mathrm{m}$-thick sections. Hematoxylin and eosin (H\&E) staining was used to observe pathological alterations. Inflammatory cell infiltration, including macrophages and neutrophils, was determined with anti-CD68 (Abcam, USA) and anti-MPO (Abcam, USA) immunohistochemical staining, respectively. To show the expression of Toll-like receptor 4 (TLR4), lung sections were incubated with primary antibody against TLR4 (Abcam, USA). Endogenous peroxides were blocked with $3 \% \mathrm{H}_{2} \mathrm{O}_{2}$, and antigen retrieval was performed by microwave heating post deparaffination and rehydration. Sections were incubated with the primary antibody (1:200) after blocking with $5 \%$ bovine serum albumin (BSA) for $30 \mathrm{~min}$. Then, the specimens were stained using a Strept Avidin-Biotin Complex (SABC) kit (Beyotime, China) according to the 
instructions. Stained sections were photographed using an Olympus inverted microscope (Olympus Imaging America, Center Valley, PA). The percentage of CD68- or MPO-positive cells per field was calculated based on 6-8 random fields.

\section{Quantitative real-time PCR (qPCR)}

Total RNA was extracted from GFP-ADSCs, IL-35-ADSCs, lung, spleen and peripheral blood samples using Trizol reagent (Invitrogen, USA) and 2- $\mu$ g RNA was reverse-transcribed using GoScript ${ }^{\mathrm{TM}}$ Reverse Transcription Mix, and an Oligo (dT) kit (Promega) according to the manufacturer's instructions. To evaluate the expression of the involved genes, qPCR detection was undertaken with a SYBR Green mix kit (Qiagen, USA). GFP expression of different tissues was used to track the infused stem cells under transgenic modification. All primers were synthesized from Sangon Biotech Co., Ltd. (Shanghai, China), and the sequences are listed in Table 1. Each sample was evaluated in triplicate. The $\Delta \Delta C_{\mathrm{t}}$ method was used for data analysis, and the level of glyceraldehyde-3-phosphate dehydrogenase (Gapdh) served as the endogenous control.

\section{Statistical analysis}

Statistical analysis was performed using SPSS version 17.0 software (SPSS Inc., Chicago, USA), and the data are presented as the mean \pm SEM. For the in vitro studies, $n=3$ for each group, and for the in vivo studies, $n=6$ for each group. An independent sample $t$ test was utilized for comparisons between two groups. One-way ANOVA was used for comparisons among four groups, and a post hoc contrast analysis using a least significant difference (LSD) $t$ test was applied to confirm the significance of differences. Two-tailed $P$ values $<0.05$ were considered statistically significant.

\section{Results}

\section{Identification of IL-35-ADSCs in vitro and the dislocation of infused transgenic cells in vivo}

The gene fragment arrangement of the plasmid pMSCVIL35-GFP is shown in Fig. 1a. As previously reported by our team (Wang et al. 2018a), ADSCs at the 3rd passage expressed a high level of CD29 (99.3\%), CD44 (99.2\%) and CD90.1 (92.3\%), but few cells expressed CD45 (2.27\%) (Fig. 1b). A fluorescent image of ADSCs $48 \mathrm{~h}$ post IL-35 modification is presented in Fig. 1c, and the transfection efficiency was $38.36 \%$. ADSCs infected by control retrovirus (GFP-ADSCs) presented similar results (Data not shown). The expression of exogenous IL-35 was confirmed via qPCR, and both Ebi3 and $I l 12 a$ were remarkably upregulated in IL-35-ADSCs (Fig. 1d). Compared with GFPADSCs, the IL-10 secretion of IL-35-ADSCs was significantly increased (Fig. 1e), suggesting augmented paracrine function post IL-35 modification, which was consistent with a previous study (Guo et al. 2017). The GFP expression was used to track the infused GFP-ADSCs or IL-35-ADSCs, and the result indicated that much more GFP signals located in lungs and spleens rather than peripheral blood (Fig. 1f), reflecting the dislocation of the injected ADSCs from one aspect. We speculated that the systemically pre-injected stem cells resided in the tissues or organs as the blood circulation.

\section{IL-35-ADSCs pretreatment attenuated LPS-induced pulmonary injury}

The experimental design for the in vivo study is shown in Fig. 2a. Mice in the saline + LPS group exhibited significant body weight loss at Day 1; while, GFP-ADSCs or IL-35-ADSCs pretreatment reversed the weight reduction (Fig. 2b, left). Compared to the saline + LPS group, stem cell administration effectively reduced the wet/dry weight

Table 1 Primer sequences for qPCR detection

\begin{tabular}{llll}
\hline Genes & Sense primers $\left(5^{\prime} \rightarrow 3^{\prime}\right)$ & Antisense primers $\left(5^{\prime} \rightarrow 3^{\prime}\right)$ & $\begin{array}{l}\text { Product } \\
\text { length } \\
(\mathrm{bp})\end{array}$ \\
\hline Ebi3 & GTT CTC CAC GGT GCC CTA C & CGG CTT GAT GAT TCG CTC & 100 \\
$I l l 2 a$ & CCA CCC TTG CCC TCC TAA A & GCC GTC TTC ACC ATG TCA TCT & 121 \\
$I l 6$ & CCA CTT CAC AAG TCG GAG GCT TA & GCA AGT GCA TCA TCG TTG TTC ATA C & 112 \\
Tnfa & AGA CAA TAA CTG CAC CCA CTT CC & ATC ATT TCC GAT AAG GCT TGG & 183 \\
$I l 10$ & GCC TTA TCG GAA ATG ATC CA & TGA GGG TCT TCA GCT TCT CAC & 115 \\
Gapdh & CAT CAC TGC CAC CCA GAA GAC TG & ATG CCA GTG AGC TTC CCG TTC AG & 153 \\
\hline
\end{tabular}

Tnf $\alpha$ tumor necrosis factor $\alpha$, Gapdh glyceraldehyde-3-phosphate dehydrogenase 
A

\begin{tabular}{|l|l|l|l|l|}
\hline EBI3 & Linker & II12a \\
\hline
\end{tabular} \begin{tabular}{|l|l|l|}
\hline IRES & GFP \\
\hline
\end{tabular}

B
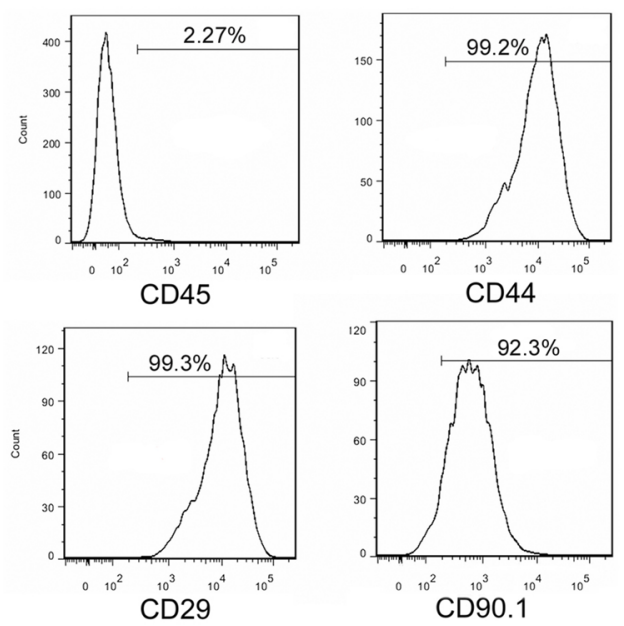

C
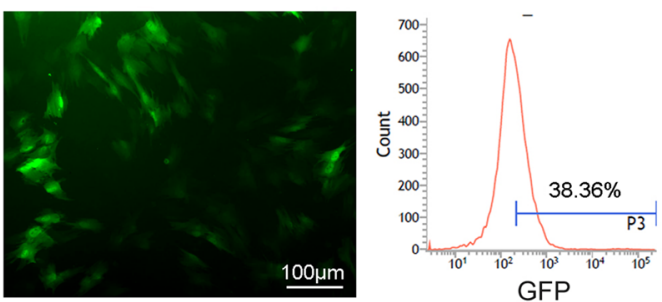

D

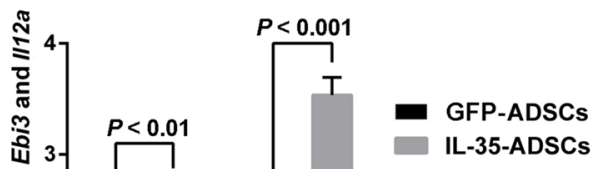

E

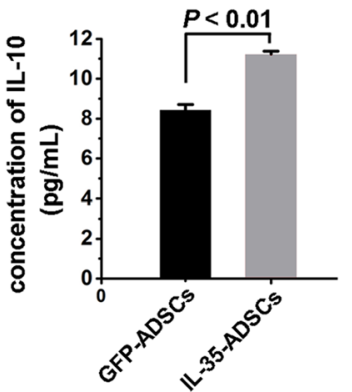

$\mathrm{F}$

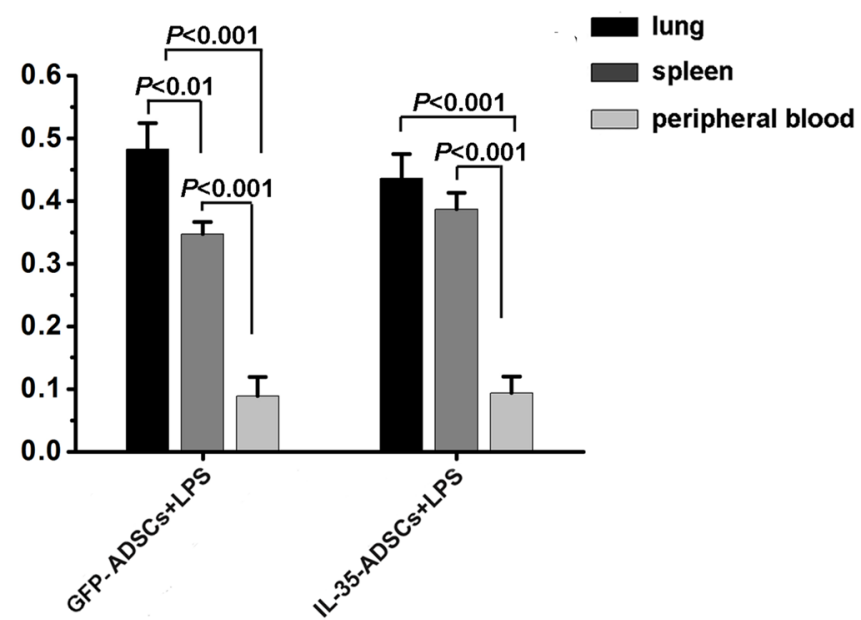

Fig. 1 Characterization of IL-35-ADSCs in vitro and tracking the infused transgenic cells in vivo. a Schematic of pMSCV-IL-35-GFP encoding the recombinant murine $I L-35$ gene consisting of EBi3 and $1112 a$. b Surface markers of 3rd passage ADSCs identified by FACS. ADSCs expressed high level of CD29, CD44 and CD90.1 but low level of CD45. c Fluorescent image of IL-35-ADSCs and the transfection efficiency determined by FACS $48 \mathrm{~h}$ post gene modification. IL-35-ADSCs were GFP positive (left). d Transcriptional expression of the two subunits of $I L-35$ in IL-35-ADSCs and GFP-ADSCs
$48 \mathrm{~h}$ after transfection. IL-35-ADSCs expressed much higher level of $E B i 3$ and $I l 12 a$ than control empty retrovirus infected ADSCs (GFPADSCs) 48 hours after transfection. $\mathrm{n}=3$ for each group. e IL-10 concentration in culture medium of IL-35-ADSCs and GFP-ADSCs $48 \mathrm{~h}$ post gene modification. $\mathrm{f}$ The GFP expression detected by qPCR reflected dislocation of infused stem cells. Much more infused cells resided in lungs and spleens rather than peripheral blood. $n=6$ for each group. Error bar: SEM 
A

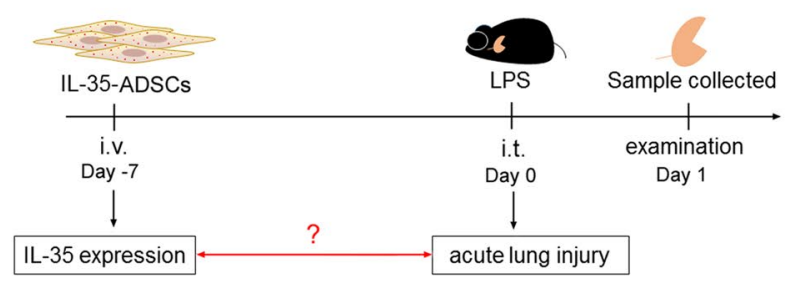

B

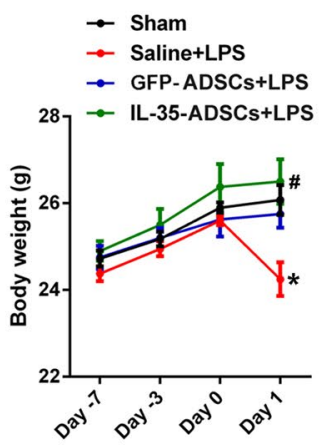

C
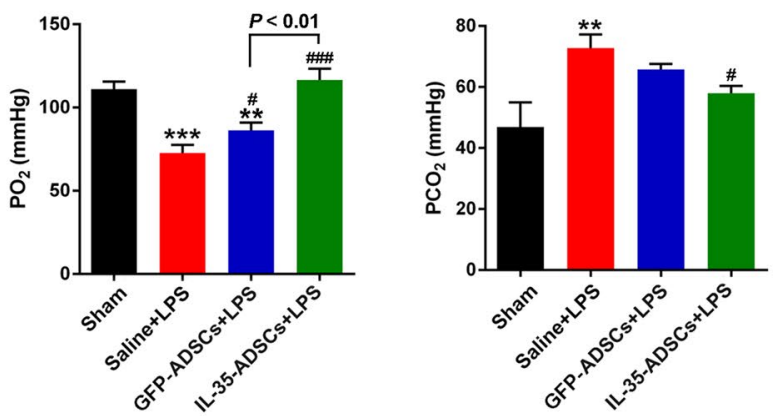

D

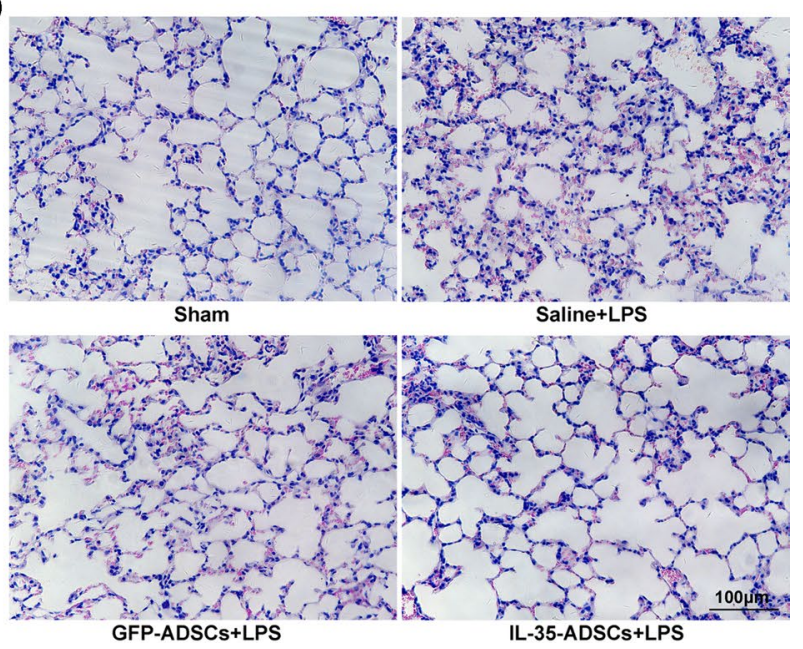

Fig. 2 IL-35-ADSCs pretreatment attenuated LPS-induced lung injury. a Experimental design for the in vivo study; i.v. intravenous injection, i.t. intratracheal inhalation. b Body weight curve and pulmonary wet/dry weight ratio of each group. Mice in the Saline + LPS group exhibited significant body weight loss and pulmonary wet/dry weight ratio increase at Day 1, while GFP-ADSCs or IL-35-ADSCs pretreatment reversed the trend. Lungs pretreated with IL-35-ADSCs had a significantly lower wet/dry weight ratio than those in GFPADSCs group. c Arterial blood $\mathrm{PO}_{2}$ and $\mathrm{PCO}_{2}$ in all mice. LPS inhalation dramatically reduced $\mathrm{PO}_{2}$ but increased $\mathrm{PCO}_{2}$. ADSCs injection relieved the ventilation disorder to some degree, and IL-

ratio of the lungs, which showed a marked increase in all LPS-exposed mice. Lungs pretreated with IL-35-ADSCs had a significantly lower wet/dry weight ratio than those in the GFP-ADSCs group (Fig. 2b, right). Blood gas assays indicated that LPS inhalation dramatically reduced $\mathrm{PO}_{2}$ but increased $\mathrm{PCO}_{2}$, suggesting damage pulmonary ventilation function. ADSCs injection relieved this trend to some degree, and IL-35-ADSCs infusion maintained the $\mathrm{PO}_{2}$ and $\mathrm{PCO}_{2}$ at normal levels (Fig. 2c).

The pulmonary pathological injury in each group was determined using H\&E staining. Lungs from mice of saline + LPS group showed severe injury characterized by diffuse alveolar hemorrhage, fluid exudation in the alveolus, alveolar damage and inflammatory cell infiltration (Fig. 2d), all of which were apparently improved by ADSCs treatment (Fig. 2d). In addition, compared with the GFP-ADSCs + LPS
35-ADSCs infusion maintained the $\mathrm{PO}_{2}$ and $\mathrm{PCO}_{2}$ at normal levels. $d$ $\mathrm{H} \& \mathrm{E}$ staining images of lungs from each group to show pathological injury. Lungs from Saline + LPS group showed severe injury characterized by diffuse alveolar hemorrhage, fluid exudation in the alveolus, alveolar damage and inflammatory cell infiltration. Compared with the GFP-ADSCs + LPS group, mice that received IL-35-ADSCs manifested much less damage, including minor hemorrhaging and infiltration of a few inflammatory cells. $\mathrm{n}=6$ for each group. Error bar: SEM. ${ }^{*} P<0.05, * * P<0.01, * * * P<0.001$ vs. Sham; ${ }^{\#} P<0.05$,

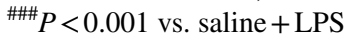

group, mice that received IL-35-ADSCs manifested much less damage, including minor hemorrhaging and infiltration of a few inflammatory cells (Fig. 2d). The above results demonstrated that IL-35-ADSCs pretreatment clearly attenuated LPS-induced ALI.

\section{IL-35-ADSCs pretreatment increased the IL-35 level but suppressed IL- 6 production, protein leakage and MPO activity in BALF}

The ELISAs to detect IL-35 showed that stem cells injection significantly increased the IL-35 concentration in BALF compared with that in the other two groups, and IL35-ADSCs pretreatment had a much higher level of IL-35 (Fig. 3a), suggesting the success of the gene modification and IL-35 overexpression in the IL-35-ADSCs + LPS group. The 
Fig. 3 IL-35-ADSCs pretreatment increased the IL-35 level but suppressed IL-6 production, protein leakage and MPO activity in BALF. a IL-35, b IL-6, and $\mathbf{c}$ total protein content and $\mathbf{d}$ MPO activity in BALF determined by ELISA. Stem cells injection significantly increased the IL-35 concentration in BALF compared with that in the other two groups, and IL-35-ADSCs pretreatment had a much higher level of IL-35. IL-6 release was remarkably elevated in all LPSchallenged mice and ADSCs pretreatment effectively inhibited IL-6 level. LPS exposure led to a significantly high level of protein exudation, which was effectively downregulated in mice that received stem cells. Pretreatment with IL-35-ADSCs rather than GFP-ADSCs rescued the protein leakage. The significantly elevated MPO level after LPS inhalation was markedly reduced by ADSCs pretreatment, and mice that received IL-35-ADSCs exhibited much lower MPO activity than those treated with GFPADSCs. $\mathrm{n}=6$ for each group. Error bar: SEM. $* P<0.05$, $* * P<0.01, * * * P<0.001$ vs. Sham; ${ }^{\#} P<0.05,{ }^{\# \#} P<0.01$, ${ }^{\# \# \# ~} P<0.001$ vs. saline + LPS
A

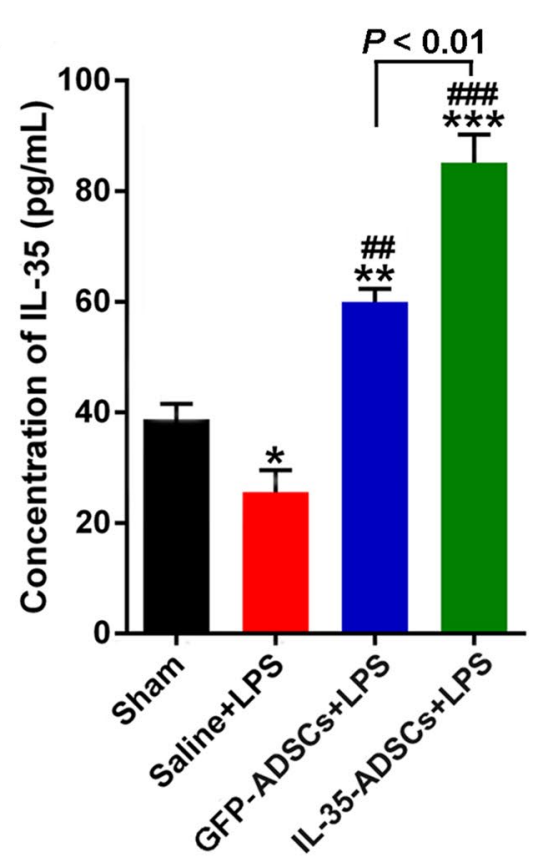

C

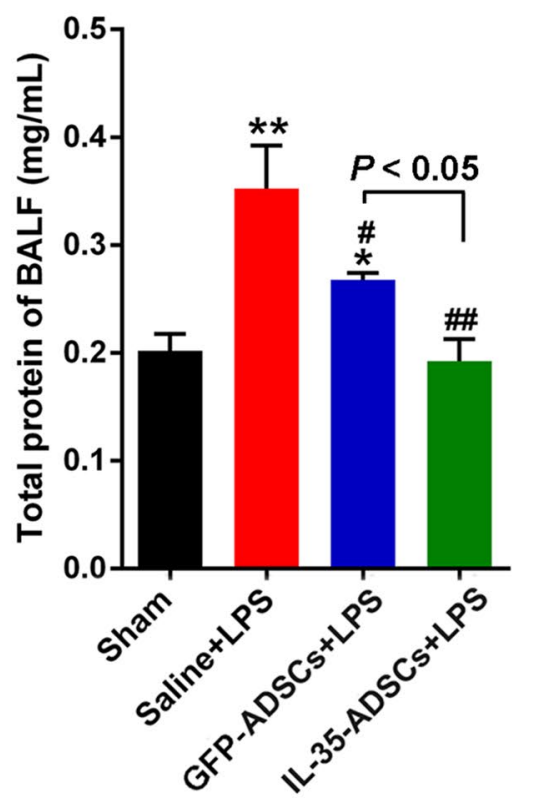

B
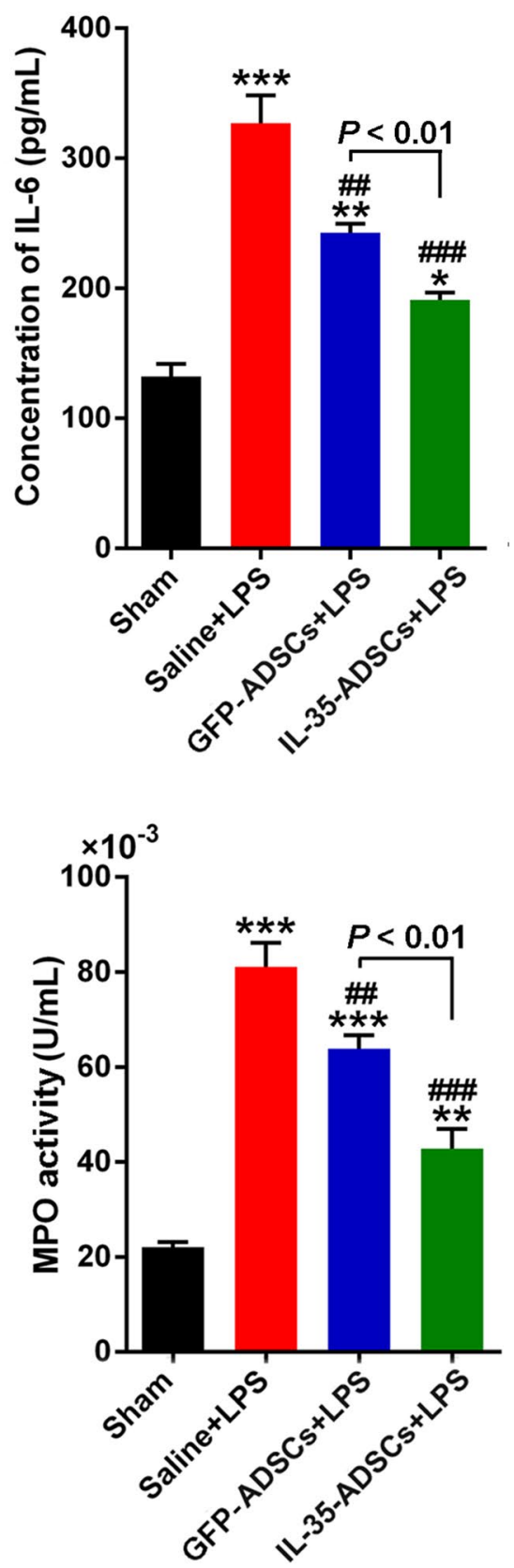

pro-inflammatory mediator IL-6, whose release was remarkably elevated in all LPS-challenged mice, got effectively inhibited (Fig. 3b). Vascular permeability increase is a hallmark of ALI, and can be reflected by the protein content in BALF. LPS exposure led to a significantly high level of protein exudation, which was effectively downregulated in mice that received stem cells (Fig. 3c). Pretreatment with IL-35-ADSCs rather than GFP-ADSCs rescued the protein leakage (Fig. 3c). In addition, significantly elevated MPO activity was observed in all LPS-exposed mice (Fig. 3d), indicating a neutrophil-mediated inflammatory response. The MPO level was markedly reduced by $\mathrm{ADSC}$ pretreatment, and mice that received IL35-ADSCs exhibited much lower MPO activity than those treated with GFP-ADSCs (Fig. 3d). The data presented here demonstrated that IL-35-ADSCs pretreatment facilitated pulmonary inflammation improvement and injury suppression.

\section{IL-35-ADSCs pretreatment significantly augmented splenic Treg cells}

IL-35, secreted by natural regulatory $\mathrm{T}$ cells (Tregs), can induce production of $\mathrm{CD} 4^{+} \mathrm{CD} 25^{+}$Tregs, whose inhibitory 
action is independent of Foxp3 expression (Castellani et al. 2010; Collison et al. 2010). In our study, to determine the effect of IL-35 expressed ADSCs on the splenic Tregs, the splenic $\mathrm{CD} 4{ }^{+} \mathrm{CD} 25^{+}$Tregs were determined in all mice using FACS (Fig. 4a). Compared with sham and saline + LPS groups, stem cells infusion significantly promoted Tregs amplification (Fig. 4b). Mice pretreated by IL35-ADSCs had the largest Tregs population, much more than those in GFP-ADSCs + LPS group (Fig. 4b). The markedly increased Tregs might be associated with the location of infused cells and assist in inflammation suppression.

\section{IL-35-ADSCs pretreatment balanced pulmonary pro-inflammatory and anti-inflammatory cytokine expression}

Cytokines are important players in the response to the inflammatory stimulation. The mRNA level of the proinflammatory cytokines $I l 6$ and Tnfo, and the anti-inflammatory cytokines $I l 10$ and $I l 35$ in the lungs were determined via $\mathrm{qPCR}$. Significantly increased $I l 6$ and Tnfo expression combined with remarkably decreased $I l 10$ and Il35 levels were observed in ALI mice treated with saline (Fig. 5a-c), indicating unbalanced cytokine production. IL-35-ADSCs pretreatment performed much better in reversing this tendency than GFP-ADSCs infusion (Fig. 5a-c). This balanced cytokine profile could facilitate improvement of the pulmonary inflammation. Moreover, the high level of Il35 expression largely depended on the pulmonary residing IL-35-ADSCs.

\section{IL-35-ADSCs pretreatment effectively inhibited inflammatory cell infiltration}

Recruitment of inflammatory cells, especially neutrophils, into pulmonary interstitium and alveolar cavities is a typical event in the acute phase of ALI (Wilhelmsen et al. 2012; Williams and Chambers 2014). Indeed, a large number of $\mathrm{MPO}^{+}$neutrophils and $\mathrm{CD}^{+} 8^{+}$macrophages were located in LPS-challenged lungs (Fig. 6). Much fewer inflammatory cells were observed in ADSCs-treated mice than those in saline + LPS group mice (Fig. 6b, d). Besides, compared to GFP-ADSCs, IL-35-ADSCs injection dramatically reduced
A

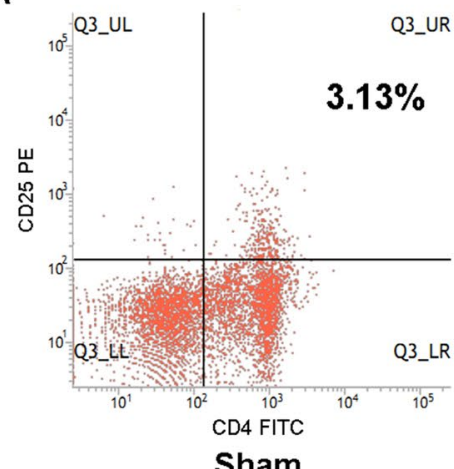

Sham

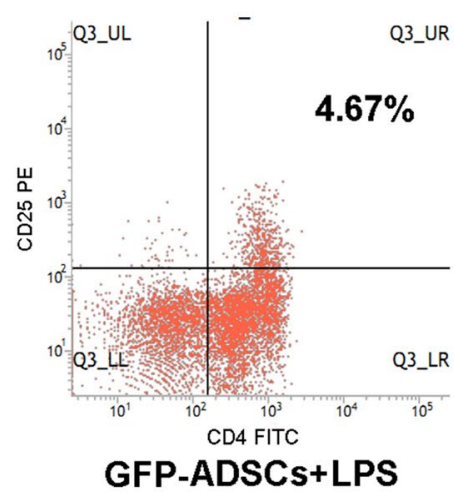

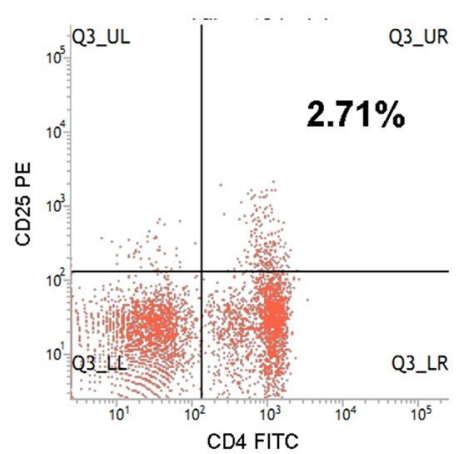

Saline+LPS

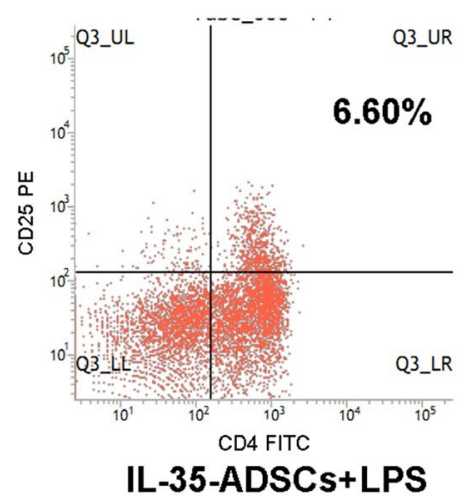

B

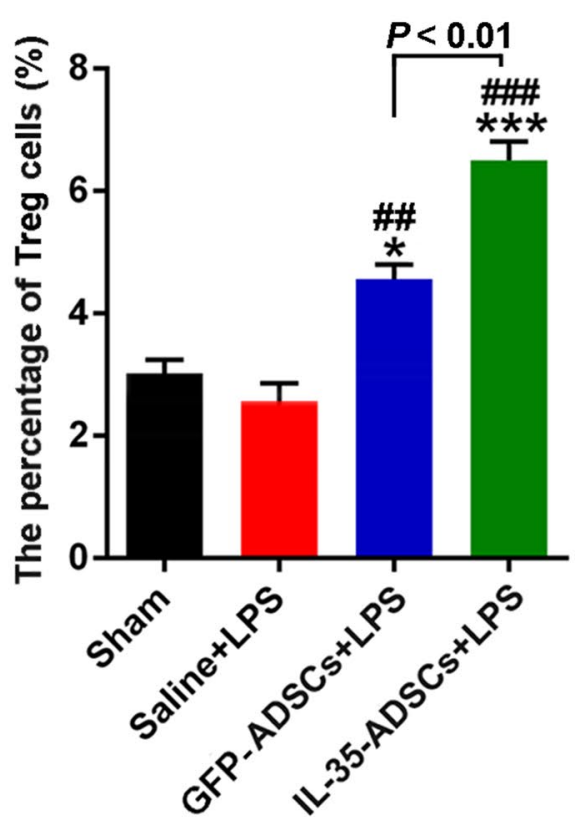

Fig. 4 IL-35-ADSCs pretreatment significantly augmented splenic Treg cells. a Representative pictures of each group showed FACS detection of $\mathrm{CD} 4^{+} \mathrm{CD} 25^{+}$Tregs in spleens. b Statistical analysis of splenic Tregs in each group. Compared with Sham and Saline + LPS groups, stem cells infusion significantly promoted Tregs ampli- fication. Mice pretreated by IL-35-ADSCs had the largest Tregs population, much more than those in LPS + GFP-ADSCs group. $\mathrm{n}=6$ for each group. Error bar: SEM. $* P<0.05, * * * P<0.001$ vs. Sham; ${ }^{\# \#} P<0.01,{ }^{\# \# \#} P<0.001$ vs. saline + LPS 
A

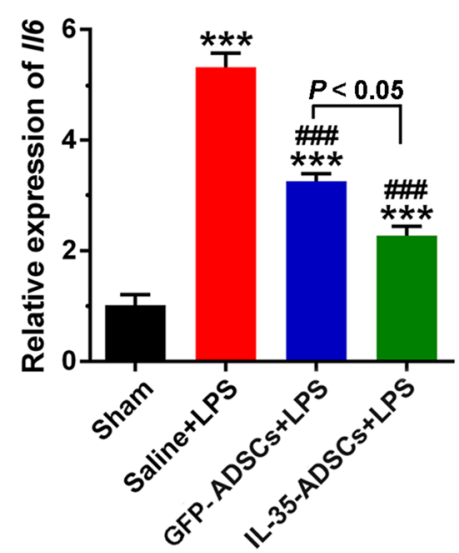

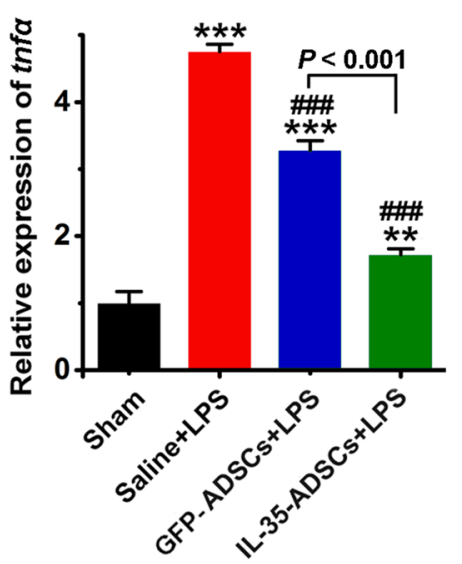

B

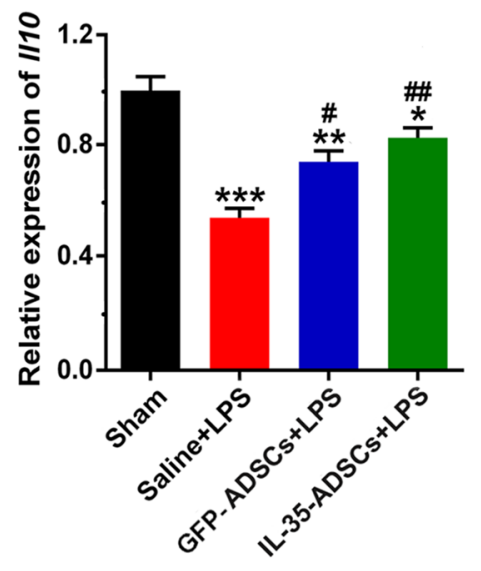

\section{C}

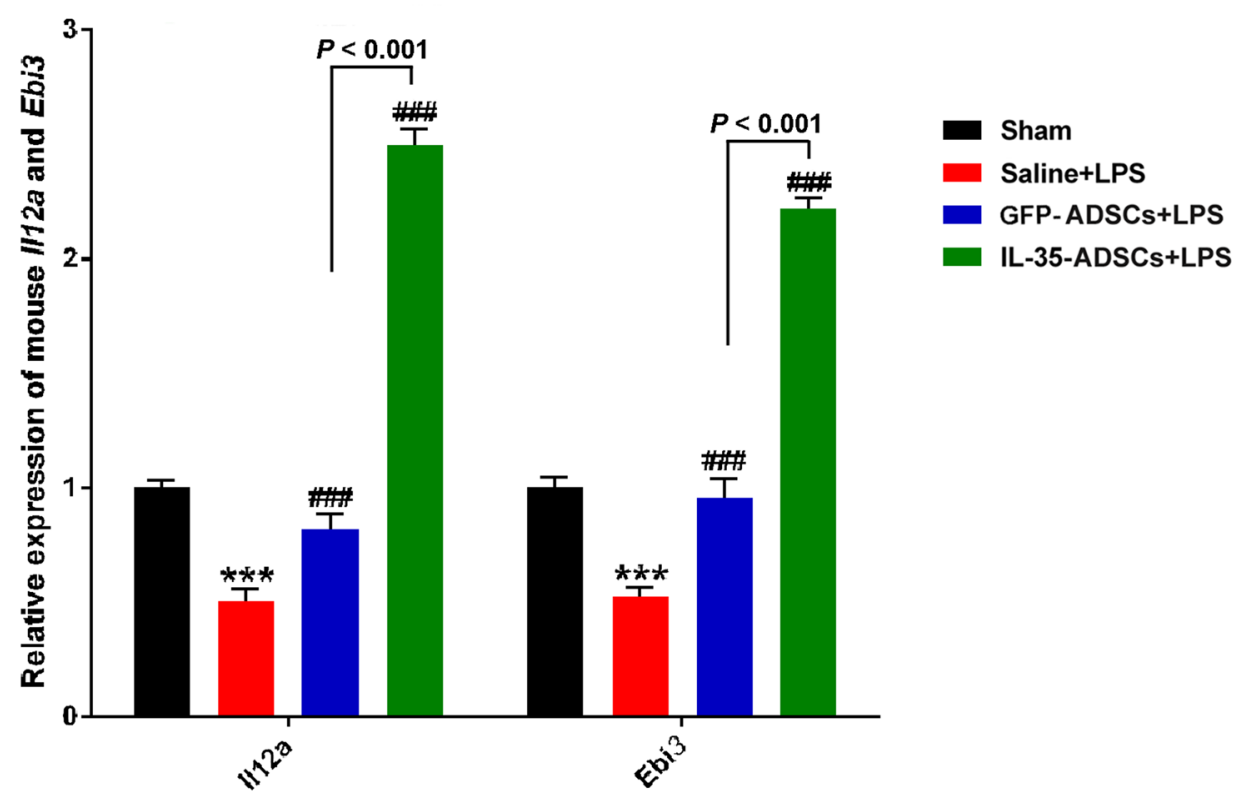

Fig. 5 IL-35-ADSCs pretreatment balanced pulmonary pro-inflammatory and anti-inflammatory cytokine expression. Pulmonary expression of a Il6 and Tnfo, b IllO and c EBi3 and Ill2a measured by qPCR. ALI mice treated by saline showed remarkably increased Il6 and Tnfo expression but reduced $I l 10$ and Il35 level due to LPS exposure. ADSCs pretreatment effectively inhibited the unbalanced cytokine profiles and IL-35-ADSCs infusion performed much better than that of GFP-ADSCs. $\mathrm{n}=6$ for each group. Error bar: SEM. ${ }^{*} P<0.05, * * P<0.01, * * * P<0.001$ vs. Sham; ${ }^{\#} P<0.05$, ${ }^{\# \#} P<0.01,{ }^{\# \# \#} P<0.001$ vs. saline + LPS neutrophil and macrophage aggregation (Fig. 6b, d), suggesting largely weakened pulmonary inflammation.

\section{IL-35-ADSCs pretreatment decreased pulmonary TLR4 expression}

Toll-like receptor 4 (TLR4), which can specifically recognize invasive LPS, has been confirmed to be closely related to the inflammatory process in pulmonary injury ( $\mathrm{Li}$ et al. 2016; Wang et al. 2016). It is also an important functional biomarker of local inflammation. We examined the TLR4 expression and found that a high TLR4 level was present in all LPS-modeled lungs (Fig. 7). IL-35-ADSCs infusion greatly lessened TLR4 expression compared with that in the GFP-ADSCs + LPS group (Fig. 7), which was beneficial to suppression of the inflammatory response in the lungs.

\section{Discussion}

ALI/acute respiratory distress syndrome (ARDS) represents a spectrum of lung diseases characterized by acute hypoxemia and severe impairment in gas exchange and lung mechanics (Matthay et al. 2012; Ware and Matthay 2000). 


\section{A}
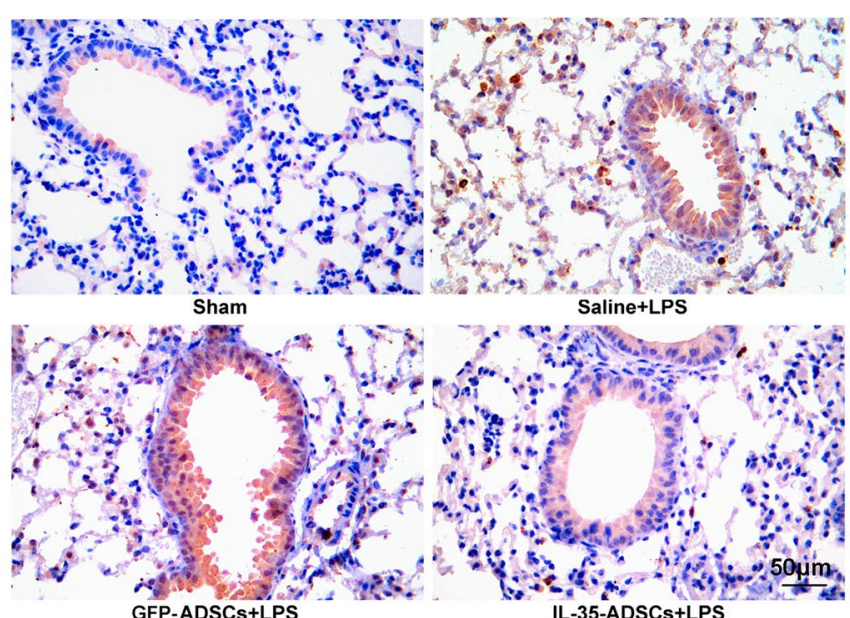

C

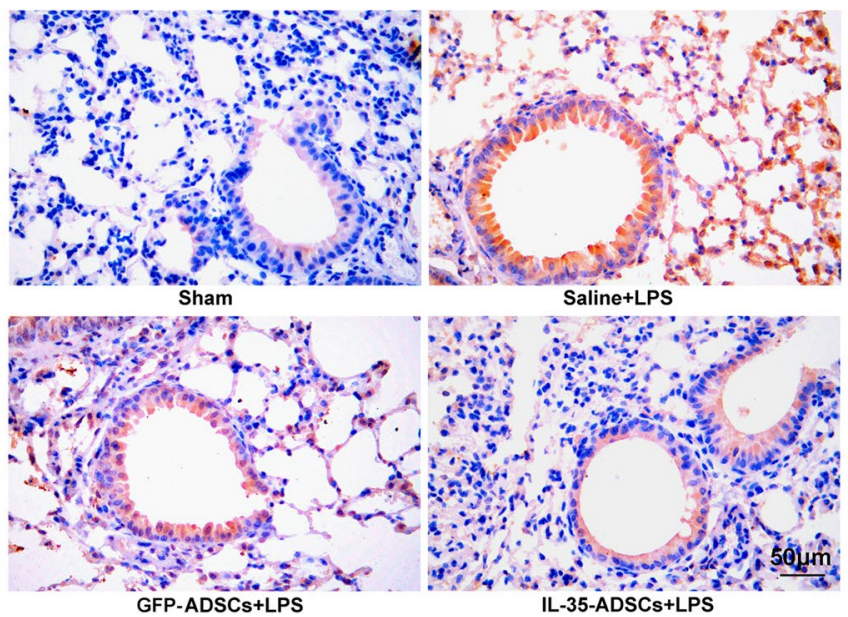

B
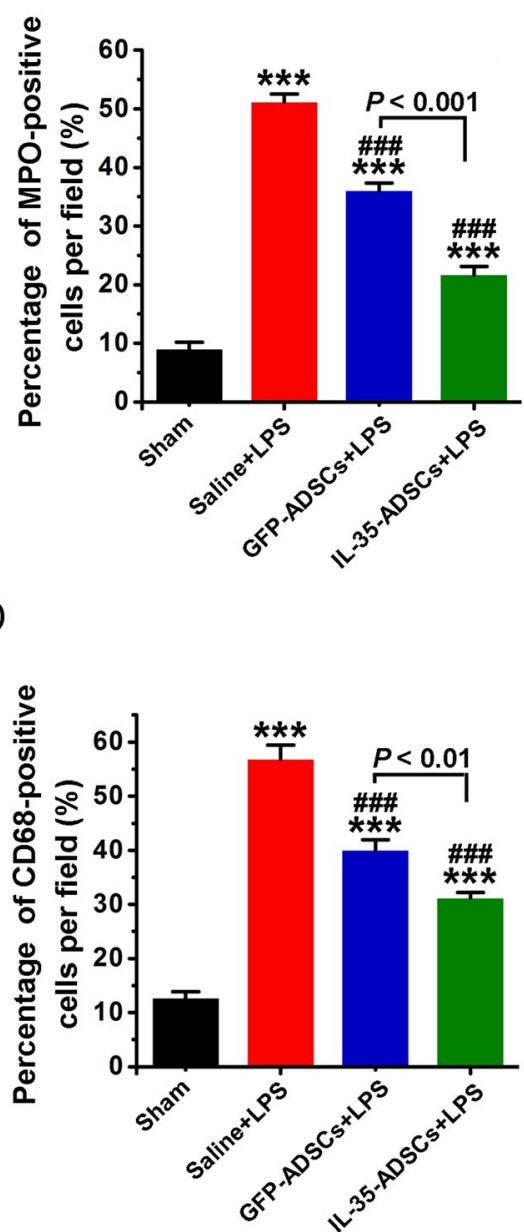

Fig. 6 IL-35-ADSCs pretreatment effectively inhibited inflammatory cell infiltration. Representative images of a MPO and c CD68 immunohistochemical staining to show the infiltrated neutrophils and macrophages in lungs from each group. The percentage of $\mathbf{b}$ MPOpositive and d CD-68-positive cells per field was calculated. A large number of $\mathrm{MPO}^{+}$neutrophils and $\mathrm{CD}^{+} 8^{+}$macrophages were located

A variety of risk factors can lead to ALI/ARDS, but the pathological process is universal. Generally, ALI/ARDS originates from uncontrolled inflammation, which leads to diffuse alveolar epithelial and vascular endothelial damage, alveolar capillary leakage, protein-rich pulmonary edema and ventilation disorder ( $\mathrm{Li}$ et al. 2016; Lin et al. 2018). Targeting the initial inflammatory process can greatly facilitate ALI/ARDS therapy.

Endotoxin-induced ALI/ARDS animal models which can provide similar inflammatory response to early process of clinical manifestations have been widely used in mechanism exploration and therapeutic expanding. Many studies have demonstrated that the inflammatory stimulation in early ALI involves inflammatory cells, pro-inflammatory and antiinflammatory cytokines (Lin et al. 2018). In the early stage in LPS-challenged lungs and ADSCs infusion effectively reduced the infiltrated inflammatory cells. Compared to GFP-ADSCs, IL-35-ADSCs injection dramatically reduced neutrophil and macrophage aggregation. $\mathrm{n}=6$ for each group. Error bar: SEM. $* * * P$ vs. Sham $<0.001$;

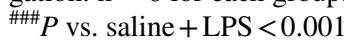

of ALI, alveolar inflammation is primarily mediated by neutrophils, whose aggregation can be found several hours post ALI (Grommes and Soehnlein 2011; Lin et al. 2018; Williams and Chambers 2014). Macrophages are also involved in the acute phase of ALI (Henderson et al. 2003; Marriott and Dockrell 2007). Besides inflammatory cell infiltration, a variety of pro-inflammatory factors including IL-6 and TNF- $\alpha$ contribute to inflammatory cell recruitment and inflammation propagation (Chen et al. 2019; Matthay et al. 2012). Meanwhile, impairment of some anti-inflammatory mediators, such as IL-10 and IL-35, aggravates the pathological injury (Wang et al. 2018a, 2019).

Good progress in ALI treatment has been made using genetically modified mesenchymal stem cells (MSCs), whose therapeutic effects are largely ascribed to their 

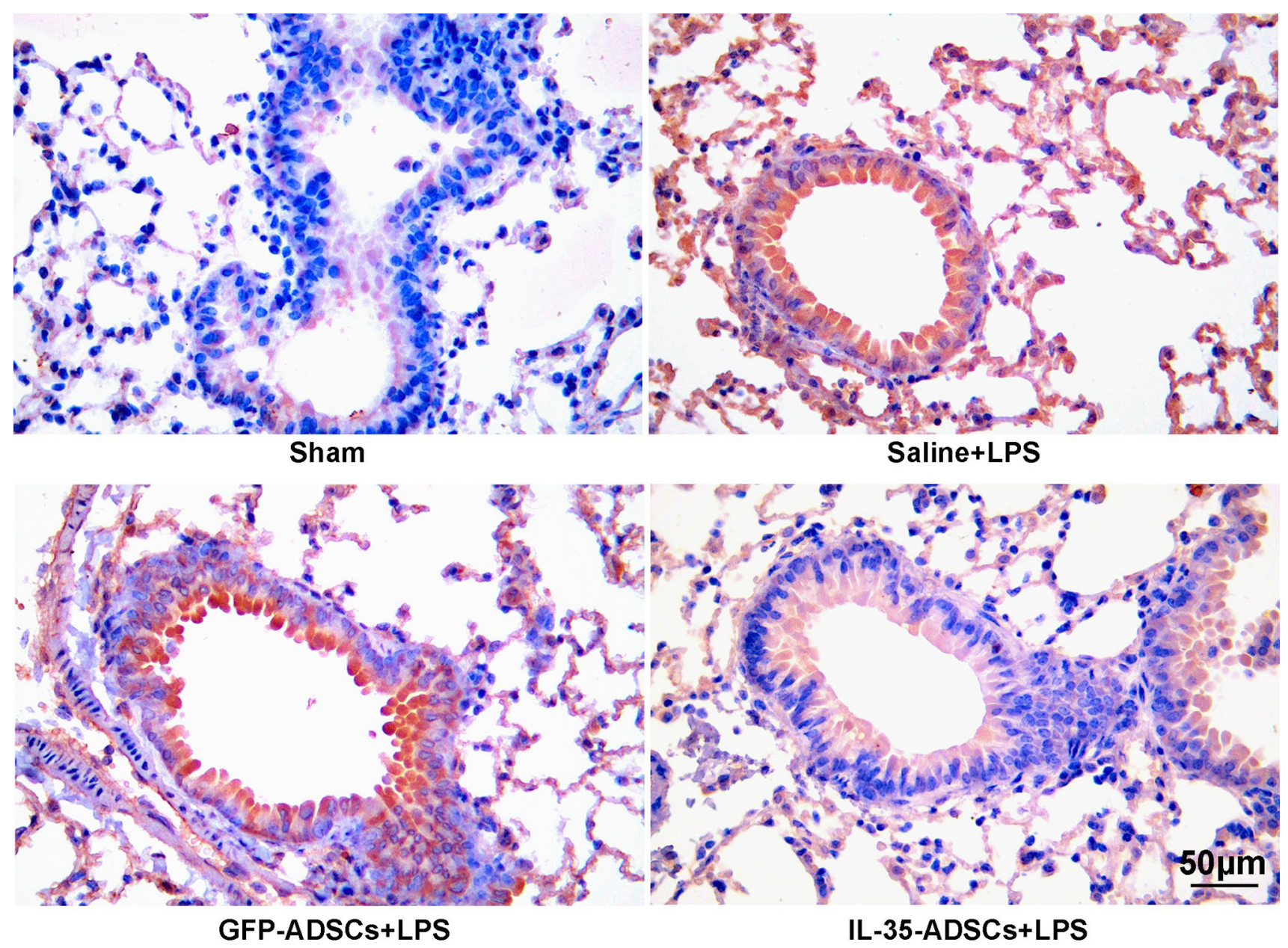

Fig. 7 IL-35-ADSCs pretreatment decreased pulmonary TLR4 expression. Immunohistochemical staining of TLR4 in lung sections from each group. A high TLR4 expression was present in all LPS-modeled lungs and IL-35-ADSCs pretreatment inhibited pulmonary TLR4 level

augmented immunomodulation and paracrine properties (Chen et al. 2018; Wang et al. 2018a). Modification with the newly identified inhibitory cytokine IL-35 can also enhance the immunosuppressive effects of adipose-derived mesenchymal stem cells (ADSCs) (Guo et al. 2017), making IL-35-engineered ADSCs (IL-35-ADSCs) a novel promising candidate for ALI intervention. The present study is the first to evaluate the protective effect of IL-35-ADSCs on LPS-induced ALI.

ADSCs were modified with retrovirus carrying the IL-35 gene and then intravenously infused into mice 7 days before ALI modeling. The level of both the anti-inflammatory cytokines IL-35 and IL-10 was apparently elevated in IL35-ADSCs, suggesting heightened paracrine action. Since intravenously delivered MSCs tend to be arrested in the lungs during circulation and have the ability to migrate and home to sites suffering injury (Soliman et al. 2018; Tian et al. 2015), pretreatment with IL-35-ADSCs might confer better protection to the lungs. Here, we first studied the protective effect of systemically pre-infused IL-35-ADSCs on the acute phase of LPS-induced ALI animals (Fig. 2a).

Data showed that much more GFP signals located in lungs and spleens instead of peripheral blood (Fig. 1f), indicating the dislocation of injected stem cells. Mice in the saline + LPS group exhibited typical symptoms of ALI, including weight loss, a significant increase in pulmonary wet/dry weight ratio, damaged ventilation function and severe pathological injury (Fig. 2b-d). ADSCs pretreatment relieved these manifestations to different degrees. Compared with GFP-ADSCs treatment, mice that received IL-35-ADSCs showed a markedly reduced pulmonary wet/dry weight ratio (Fig. 2b) and BALF protein content (Fig. 3c), suggesting well-controlled pulmonary edema and protein leakage. The $\mathrm{PO}_{2}$ in IL-35-ADSCs-treated mice reached normal levels and was much higher than that in GFP-ADSCs + LPS mice (Fig. 2c). Alveolar hemorrhage, fluid exudation and inflammatory cell infiltration in the lungs were also noticeably attenuated (Fig. 2d). 
The pulmonary level of IL-10 and IL-35 both showed a significant increase $24 \mathrm{~h}$ post ALI exposure (Fig. 3a). As reliable biomarkers of ALI animals or patients, pulmonary IL- 6 and TNF- $\alpha$ were also most effectively inhibited in mice that received IL-35-ADSCs (Fig. 5a). Similar to the $\mathrm{H} \& \mathrm{E}$ staining results, many more $\mathrm{MPO}^{+}$neutrophils and $\mathrm{CD}^{2} 8^{+}$macrophages could be observed in the lungs from saline + LPS mice, and ADSCs injection effectively inhibited these infiltrates (Fig. 6a). Lungs treated with IL-35-ADSCs exhibited significantly decreased neutrophil and macrophage accumulation compared with that in GFP-ADSCs + LPS group lungs (Fig. 6b). In addition, IL-35-ADSCs infusion greatly suppressed TLR4 expression compared with that in GFP-ADSC-treated mice (Fig. 7). These data indicate that IL-35-ADSCs pretreatment effectively attenuated local inflammation in LPS-challenged lungs.

Natural regulatory T cells (Tregs) have important inhibitory properties that largely rely on IL-35 expression, and IL-35 can induce Treg production and proliferation (Chaturvedi et al. 2013; Collison et al. 2010). Previous research has demonstrated that Tregs can protect lungs from inflammatory injury (Lin et al. 2018; Wang et al. 2019). Compared with GFP-ADSCs + LPS group mice, the number of splenic $\mathrm{CD} 4{ }^{+} \mathrm{CD} 25^{+}$Tregs was remarkably amplified in IL35-ADSCs-treated mice, primarily due to the elevated IL-35 level (Fig. 4). It has been reported that Th17 cells-mediated inflammation also participate in the inflammatory process of ALI, and the unbalanced Tregs/Th17 cells often exist in ALI animals or patients (Wang et al. 2018b; Zhang et al. 2016). Reversed Tregs/Th17 cells contribute to amelioration of the damage induced by ALI. The Th17 cells was not detected here, but we have found that IL-35 upregulation significantly increased $\mathrm{CD} 4{ }^{+} \mathrm{CD} 25^{+} \mathrm{Foxp} 3^{+}$Treg cells and reduced IL-17 $\mathrm{A}^{+}$Th17 cells of the spleens and mesenteric lymph nodes in dextran sulfate sodium (DSS)-induced colitis models (Zhang et al. 2018a). The growth of Tregs might also facilitate the ALI inhibition.

It should be noted that the benefits of IL-35-ADSCs pretreatment against acute inflammatory injury were confined to the LPS-induced ALI model. But the detail mechanism of IL-35 on pulmonary function and injury repair in long term was not confirmed. Since animal models cannot reproduce all the characteristics of ALI/ARDS in humans, the efficacy of the same treatment on another symptoms or another kinds of ALI models still needs further study.

\section{Conclusions}

In the current study, the protective efficacy of pretreatment with IL-35-engineered ADSCs in LPS-induced ALI was evaluated. The data indicated that IL-35-ADSCs administration greatly attenuated the inflammatory damage in the lungs and, thus, might provide an alternative strategy for ALI intervention. The pulmonary protective effects of pretreatment with IL-35-ADSCs might be mainly attributed to their enhanced paracrine and immunosuppressive function. Additional investigations are still needed to elucidate the underlying mechanism.

Acknowledgements The present work was supported by the grants from National Key R\&D Program of China (2017YFC1001900 and 2017YFC1001904), Tianjin Science and Technology Support Program (16YFZCSY00900), National Natural Science Foundation of China for Young Scholars (81501386, 81601047 and 81600073), Tianjin Medical University 13th 5-year postgraduate Innovation Fund (YJSCX201705), Academic Promotion Programme of Shandong First Medical University and The Innovation Project of Shandong Academy of Medical Sciences.

Author contributions XNZ, GL, and YL designed and analyzed the data, XNZ, ZQZ, MYJ, JCL, YQJ, YXZ, CG and MD carried out the experiments, XNZ and YL wrote the manuscript. All authors reviewed the manuscript.

\section{Compliance with ethical standards}

Conflict of interest The authors have no conflict of interests.

\section{References}

Bellani G et al (2016) Epidemiology, patterns of care, and mortality for patients with acute respiratory distress syndrome in intensive care units in 50 countries. JAMA J Am Med Assoc 315:788-800. https://doi.org/10.1001/jama.2016.0291

Castellani ML et al (2010) IL-35, an anti-inflammatory cytokine which expands CD4+CD25+ Treg cells. J Biol Regul Homeost Agents 24:131-135

Chaturvedi V, Collison LW, Guy CS, Workman CJ, Vignali DA (2013) Retraction. Human regulatory T cells require IL-35 to mediate suppression and infectious tolerance. J Immunol 191:2018. https ://doi.org/10.4049/jimmunol.1390040

Chen XX, Zhang YL, Wang WJ, Liu ZQ, Meng JG, Han ZH (2018) Mesenchymal stem cells modified with heme oxygenase- 1 have enhanced paracrine function and attenuate lipopolysaccharideinduced inflammatory and oxidative damage in pulmonary microvascular endothelial cells. Cell Physiol Biochem 49:101-122. https://doi.org/10.1159/000492847

Chen X et al (2019) Mesenchymal stem cells overexpressing heme oxygenase-1 ameliorate lipopolysaccharide-induced acute lung injury in rats. J Cell Physiol 234:7301-7319. https://doi.org/10.1002/ jcp. 27488

Cho YJ et al (2016) Clinical practice guideline of acute respiratory distress syndrome. Tuberc Respir Dis 79:214-233. https://doi. org/10.4046/trd.2016.79.4.214

Cobbold SP et al (2003) Regulatory T cells and dendritic cells in transplantation tolerance: molecular markers and mechanisms. Immunol Rev 196:109-124

Collison LW et al (2007) The inhibitory cytokine IL-35 contributes to regulatory T-cell function. Nature 450:566-569. https://doi. org/10.1038/nature06306

Collison LW et al (2010) IL-35-mediated induction of a potent regulatory T cell population. Nat Immunol 11:1093-1101. https://doi. org/10.1038/ni.1952 
D’Alessio FR et al (2009) CD4(+)CD25(+)Foxp3(+) Tregs resolve experimental lung injury in mice and are present in humans with acute lung injury. J Clin Investig 119:2898-2913. https://doi. org/10.1172/JCI36498

Fanelli V, Ranieri VM (2015) Mechanisms and clinical consequences of acute lung injury. Ann Am Thorac Soc 12(Suppl 1):S3-8. https ://doi.org/10.1513/AnnalsATS.201407-340MG

Grommes J, Soehnlein O (2011) Contribution of neutrophils to acute lung injury. Mol Med 17:293-307. https://doi.org/10.2119/molme d. 2010.00138

Guo H, Zhao N, Gao H, He X (2017) Mesenchymal stem cells overexpressing interleukin-35 propagate immunosuppressive effects in mice. Scand J Immunol 86:389-395. https://doi.org/10.1111/ sji. 12613

Henderson RB, Hobbs JAR, Mathies M, Hogg N (2003) Rapid recruitment of inflammatory monocytes is independent of neutrophil migration. Blood 102:328-335. https://doi.org/10.1182/blood $-2002-10-3228$

Kuo J, Nardelli DT, Warner TF, Callister SM, Schell RF (2011) Interleukin-35 enhances Lyme arthritis in Borrelia-vaccinated and -infected mice. Clin Vaccine Immunol 18:1125-1132. https:// doi.org/10.1128/CVI.00052-11

Li DQ et al (2016) Bone marrow mesenchymal stem cells suppress acute lung injury induced by lipopolysaccharide through inhibiting the Tlr 2, 4/Nf-kappa B pathway in rats with multiple trauma. Shock 45:641-646. https://doi.org/10.1097/Shk.0000000000 000548

Lin SH, Wu H, Wang CJ, Xiao ZB, Xu F (2018) Regulatory T cells and acute lung injury: cytokines, uncontrolled inflammation, and therapeutic implications. Front Immunol. https://doi.org/10.3389/ Fimmu.2018.01545

Maddaloni M, Kochetkova I, Hoffman C, Pascual DW (2018) Delivery of IL-35 by Lactococcus lactis ameliorates collagen-induced arthritis in mice. Front Immunol. https://doi.org/10.3389/Fimmu .2018 .02691

Manabe T et al (2018) Clinical outcomesin patients with acute respiratory distress syndrome (Ards) in Vietnam according to severity of the Berlin definition. Respirology 23:262-262

Marriott HM, Dockrell DH (2007) The role of the macrophage in lung disease mediated by bacteria. Exp Lung Res 33:493-505. https:// doi.org/10.1080/01902140701756562

Matthay MA, Ware LB, Zimmerman GA (2012) The acute respiratory distress syndrome. J Clin Investig 122:2731-2740. https:// doi.org/10.1172/JCI60331

Monge RH et al (2014) Incidence, outcomes and risk factors associated with the development of acute lung injury and at respiratory distress syndrome in patients with severe sepsis and septic shock admitted to ICU. Results of a Spanish Multicenter Study. Intens Care Med 40:S133-S133

Ozdemir RBO, Ozdemir AT, Sariboyaci AE, Uysal O, Tuglu MI, Kirmaz C (2019) The investigation of immunomodulatory effects of adipose tissue mesenchymal stem cell educated macrophages on the CD4 T cells. Immunobiology 224:585-594. https://doi. org/10.1016/j.imbio.2019.04.002

Shi Y et al (2010) Mesenchymal stem cells: a new strategy for immunosuppression and tissue repair. Cell Res 20:510-518. https://doi. org/10.1038/cr.2010.44

Soliman MG, Mansour HA, Hassan WA, El-Sayed RA, Hassaan NA (2018) Mesenchymal stem cells therapeutic potential alleviate lipopolysaccharide-induced acute lung injury in rat model. J Biochem Mol Toxic. https://doi.org/10.1002/jbt.22217
Tian WJ et al (2015) Infusion of mesenchymal stem cells protects lung transplants from cold ischemia-reperfusion injury in mice. Lung 193:85-95. https://doi.org/10.1007/s00408-014-9654-x

Wang JC, Qin Y, Mi XJ (2016) The protective effects of bone marrowderived mesenchymal stem cell (BMSC) on LPS-induced acute lung injury via TLR3-mediated IFNs, MAPK and NF-kappa B signaling pathways. Biomed Pharmacother 79:176-187. https:// doi.org/10.1016/j.biopha.2016.02.037

Wang CF, Lv D, Zhang XB, Ni ZA, Sun XF, Zhu CQ (2018a) Interleukin-10-overexpressing mesenchymal stromal cells induce a series of regulatory effects in the inflammatory system and promote the survival of endotoxin-induced acute lung injury in mice model. DNA Cell Biol 37:53-61. https://doi.org/10.1089/dna.2017.3735

Wang L et al (2018b) Recovery from acute lung injury can be regulated via modulation of regulatory T cells and Th17 cells. Scand J Immunol 88:e12715. https://doi.org/10.1111/sji.12715

Wang Y et al (2018c) IL-35 recombinant protein reverses inflammatory bowel disease and psoriasis through regulation of inflammatory cytokines and immune cells. J Cell Mol Med 22:1014-1025. https ://doi.org/10.1111/jcmm.13428

Wang CJ, Zhang M, Wu H, Lin SH, Xu F (2019) IL-35 interferes with splenic $\mathrm{T}$ cells in a clinical and experimental model of acute respiratory distress syndrome. Int Immunopharmacol 67:386-395. https://doi.org/10.1016/j.intimp.2018.12.024

Ware LB, Matthay MA (2000) The acute respiratory distress syndrome. N Engl J Med 342:1334-1349. https://doi.org/10.1056/NEJM2 00005043421806

Wilhelmsen K, Mesa KR, Prakash A, Xu F, Hellman J (2012) Activation of endothelial TLR 2 by bacterial lipoprotein upregulates proteins specific for the neutrophil response. Innate Immun 18:602-616. https://doi.org/10.1177/1753425911429336

Williams AE, Chambers RC (2014) The mercurial nature of neutrophils: still an enigma in ARDS? Am J Physiol Lung Cell Mol Physiol 306:L217-230. https://doi.org/10.1152/ajplung.00311 .2013

Yao XP et al (2015) Nitric oxide releasing hydrogel enhances the therapeutic efficacy of mesenchymal stem cells for myocardial infarction. Biomaterials 60:130-140. https://doi.org/10.1016/j.bioma terials.2015.04.046

Zhang F, Li MY, Lan YT, Wang CB (2016) Imbalance of Th17/Tregs in rats with smoke inhalation-induced acute lung injur. Sci Rep 6:21348. https://doi.org/10.1038/srep21348

Zhang B et al (2018a) Oral Escherichia coli expressing IL-35 meliorates experimental colitis in mice. J Transl Med 16:71. https://doi. org/10.1186/s12967-018-1441-7

Zhang SQ, Jiang W, Ma LJ, Liu YH, Zhang XY, Wang S (2018b) Nrf2 transfection enhances the efficacy of human amniotic mesenchymal stem cells to repair lung injury induced by lipopolysaccharide. J Cell Biochem 119:1627-1636. https://doi.org/10.1002/jcb.26322

Zhang XN et al (2018c) Interleukin 35 induced Th2 and Tregs bias under normal conditions in mice. PeerJ. https://doi.org/10.7717/ Peerj.5638

Publisher's Note Springer Nature remains neutral with regard to jurisdictional claims in published maps and institutional affiliations. 